\title{
CFD analysis and optimization of a liquid lead-bismuth loop target for ISOL facilities
}

\author{
D. Houngbo ${ }^{\mathrm{a}, \mathrm{b}, *}$, L. Popescu $^{\mathrm{a}}$, P. Schuurmans ${ }^{\mathrm{a}}$, M. Delonca ${ }^{\mathrm{c}}$, R. Losito ${ }^{\mathrm{c}}$, C. Maglioni $^{\mathrm{c}}$, \\ T. Stora ${ }^{\mathrm{c}}$, P. Bricault ${ }^{\mathrm{d}}$, J. Vierendeels ${ }^{\mathrm{b}}$ \\ a Belgian Nuclear Research Centre (SCK.CEN), Boeretang 200, B-2400 Mol, Belgium \\ ${ }^{\mathrm{b}}$ Department of Flow, Heat and Combustion Mechanics, Ghent University (UGent), St.-Pietersnieuwstraat 41, B-9000 Gent, Belgium \\ c CERN, 1211 Geneva 23, Switzerland \\ ${ }^{\mathrm{d}}$ TRIUMF, 4004 Wesbrook Mall, Vancouver, B.C., Canada V6T 2A3
}

\section{A R T I C L E I N F O}

\section{Article history:}

Received 27 August 2014

Received in revised form

11 December 2014

Accepted 13 December 2014

Available online 20 December 2014

\section{Keywords:}

Computational Fluid Dynamics

High-power target

Liquid metal

ISOL

Radioactive Ion Beams

\begin{abstract}
A B S T R A C T
In the context of the forthcoming next generation of Radioactive Ion Beams (RIBs) facilities based on an Isotope Separation On Line (ISOL) method, the development of production targets capable of dissipating the high power deposited by the primary beam is a major challenge. The concept of a high-power target based on a liquid $\mathrm{Pb}-\mathrm{Bi}$ loop incorporating a heat-exchanger and a diffusion chamber was proposed within EURISOL DS and is being developed within the LIEBE ${ }^{1}$ project. Due to the non-static character of the target, specific hydrodynamics issues are of concern. In this paper, these issues are studied mostly based on three-dimensional (3D) Computational Fluid Dynamics analysis of the flow of the Lead Bismuth Eutectic (LBE) target, resulting in optimized designs. The concept and hydrodynamic challenges of generating RIBs from a liquid-metal-loop target irradiated with a high-power primary beam are presented. The optimization of the target design has been conducted keeping in mind the need for a fast and efficient release of short-lived isotopes.

This study shows that approximately $100 \mathrm{~ms}$ after the proton pulse the irradiated liquid-metal is entirely and uniformly evacuated from the irradiation volume and spread in a shower of small droplets (100- $\mu \mathrm{m}$ radii), in order to reduce the diffusion length of isotopes. Solutions to deal with the typical cavitation risk due to the presence of low-pressure zones in the liquid have also been found and simulated.
\end{abstract}

(c) 2014 Elsevier B.V. All rights reserved.

\section{Introduction}

Applications of Radioactive Ion Beams (RIBs) are found in several fields of science, e.g. nuclear physics, fundamental interactions, and nuclear astrophysics [1]. Because of their short halflives, production of exotic nuclei using off-line separation techniques is discarded, since the isotopes would completely decay before separation. The only way to produce these beams is using on-line methods: either in-flight separation (IFS), or isotope separation on-line (ISOL) [1]. The IFS method mostly makes use of high-energy nuclear beams impacting on a thin target and relies on the recoil of the reaction products in combination with magnetic or electrical fields to separate the isotopes of interest. In the ISOL method, the isotopes of interest are produced and released from a thick target, kept at high temperature, which often

\footnotetext{
* Corresponding author at: Belgian Nuclear Research Centre (SCK•CEN), Boeretang 200, B-2400 Mol, Belgium. Tel.: +32 14333416.

E-mail address: dhoungbo@sckcen.be (D. Houngbo).

${ }^{1}$ LIquid IEad Bismuth eutectic loop target for EURISOL (LIEBE).
}

also serves as catcher to thermalize the reaction products. After release, the isotopes are subsequently ionized, extracted and mass separated.

The RIBs produced through IFS are characterized by high energy, a relatively low purity, a large energy spread and large transversal emittances. On the other hand, the ISOL beams are characterized by high purity and small transverse emittance, but, in the absence of a post-accelerator, the energies of the ions are low (30-60 keV). For exotic RIBs, the main advantage of the ISOL technique comes from the higher achievable purities, since study of the exotic nuclei is often hindered by the fact that they are submerged in the high background of more stable nuclei also produced in the target. However, it should be noted that decay losses during the isotope-release and ionization processes constitute an important drawback for the production of very short-lived isotopes (half-lives in the millisecond range) through the ISOL method.

Worldwide efforts are currently devoted to prepare a new generation of RIB facilities, with the aim of increasing the intensity of the beams by several orders of magnitude compared to presently running 
facilities [1]. The development of high-power targets is therefore a major part of this preparation since, at a given facility, RIB intensities should scale with increasing the primary-beam current. ISOL targets capable of withstanding high-power primary beams without compromising the reliability of the yields and the structural integrity of the target over extended periods of time are therefore of interest. Such targets are being developed, e.g., within the EURISOL [2] and ISOL@MYRRHA projects [3].

Different concepts for high-power targets have been proposed and studied in the framework of the preparation for the newgeneration RIB facilities. Based on radiative heat transfer studies, Zhang and Alton [4] have proposed porous and fibrous composite targets, that are expected to operate with deposited beam powers of up to $40 \mathrm{~kW}$. Besides, upon testing with both resistive and electron-beam heating, the TRIUMF-ISAC high-power target equipped with a finned container was shown to be capable of dissipating more than $25 \mathrm{~kW}$ when operated at $2200{ }^{\circ} \mathrm{C}$ [5]. However, these different studies concern solid targets, and characterized by relatively low thicknesses.

An alternative approach is the recently proposed liquid-metalloop target [6], which is expected to handle much higher primarybeam power because the target material flows in a loop equipped with a heat exchanger. In addition, liquid targets typically offer the highest thicknesses of any material $\left(200 \mathrm{~g} / \mathrm{cm}^{2}\right.$ for Lead Bismuth Eutectic (LBE)). A thicker target typically means a higher number of target atoms per unit surface being exposed to the primary beam, which leads to higher in-target production rates for the isotopes of interest. Concerns for the design of these targets include effects like pressure drop, cavitation, liquid-metal recirculation, instabilities, non-uniform flows etc. [7-9]. Studies conducted in the past by different researchers highlight the importance of these effects on the target system $[10,11]$.

The liquid-metal-loop target is one of the foreseen 100-kW EURISOL direct targets and is also of interest for the ISOL@MYRRHA facility. The study reported here aims at providing solutions to specific hydrodynamics issues of concern for the design of this target. The satisfactory concepts will be prototyped and tested at CERN-ISOLDE within the LIEBE (LIquid lEad Bismuth eutectic loop target for EURISOL) project. The design optimization based on criteria detailed in Section 2 is also presented, followed by a discussion of the hydraulic aspects of different optimized concepts.

Beam parameters considered for EURISOL, ISOL@MYRRHA and the test facility CERN-ISOLDE are given in Table 1 for reference.

\section{Target concept}

Detailed understanding of the time dependence of the release process of radioisotopes is necessary for the development of improved targets for ISOL RIB production. Liquid-lead targets have been studied and operated at ISOLDE with $600-\mathrm{MeV}$ protons from the SynchroCyclotron (SC) and later on, with $1-1.4-\mathrm{GeV}$ protons from the Proton-Synchrotron-Booster (PS-Booster) [12]. The partially-filled liquid-metal-target containers were $20-\mathrm{cm}$ long cylinders with a $1-\mathrm{cm}$ radius. At the SC, the time necessary to release half of the isotopes $\left(T_{50}\right)$ from molten-metal targets was typically from 30 to $120 \mathrm{~s}$ [13]. The violent shaking of the target, due to proton pulses from the PS-Booster, has induced faster release of isotopes. However, the $T_{50}$ calculated from fitted release function for mercury atoms was still of $10 \pm 5 \mathrm{~s}$ [13], which is long in comparison with the half-lives of short-lived isotopes like ${ }^{178} \mathrm{Hg}$ $\left(T_{1 / 2}=0.26 \mathrm{~s}\right)$ and ${ }^{177} \mathrm{Hg}\left(T_{1 / 2}=0.13 \mathrm{~s}\right)$.

The proposed concept for the liquid-metal-loop target is shown in Fig. 1. In this concept, a dedicated container, filled with LBE, is used for irradiating the target material by the proton beam. This container, called "irradiation volume", is placed in a vacuum chamber, called "diffusion volume". After irradiation by protons, LBE droplets are created, forming a shower inside the diffusion volume. The droplets fall under gravity before being collected at the bottom of the diffusion volume. Meanwhile, the isotopes diffuse to the surface of the droplets, desorb from this surface and effuse towards the ion source via the transfer tube. A heat exchanger is foreseen upstream to the pump, to ensure that the LBE temperature at the pump inlet does not exceed maximum acceptable values for the magnets of the pump $\left(600^{\circ} \mathrm{C}\right)$. The pump ensures a constant LBE flow. Heating devices foreseen along the loop allow for a better control of the temperature at start-up and during operation.

Based on the knowledge derived from operation of the staticbath liquid-metal targets and in order to improve the efficiency of the release process for short-lived isotopes, two major requirements were set prior to the detailed design of the liquid-metalloop target:

- Fast evacuation (within $100 \mathrm{~ms}$ ) of all the irradiated liquid metal from the irradiation volume to the diffusion volume.

- Formation of small and spherical liquid-metal droplets ( $\sim 100 \mu \mathrm{m}$ radii) inside the diffusion volume.

Because the objective of this target is to produce short-lived isotopes like ${ }^{177} \mathrm{Hg}\left(T_{1 / 2}=0.13 \mathrm{~s}\right)$, the first requirement is the fast evacuation of all the irradiated LBE from the irradiation volume into the diffusion volume to minimize the decay losses of these isotopes in the irradiation volume. In the present design, we aimed at a complete evacuation of the irradiated LBE in a shower of droplets within $\sim 100 \mathrm{~ms}$ after a proton-pulse impact. This requirement also

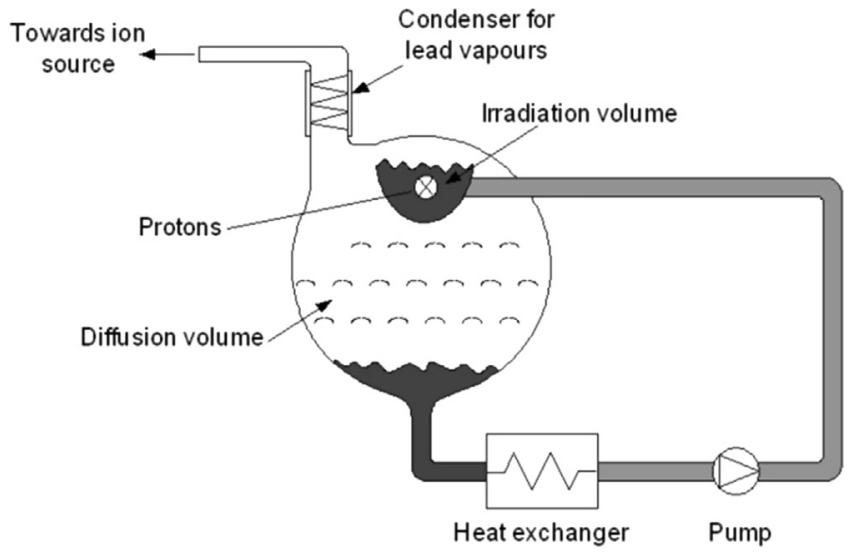

Fig. 1. Conceptual view of the target as proposed within the EURISOL Design Study [14].

Table 1

Beam parameters for EURISOL, ISOL@MYRRHA and CERN-ISOLDE facilities.

\begin{tabular}{|c|c|c|c|c|c|c|c|}
\hline & Particles & Energy (GeV) & Intensity $(\mu \mathrm{A})$ & Beam structure & Pulse length & Repetition rate & Average power $(\mathrm{kW})$ \\
\hline EURISOL & Protons & 1 & 100 & $\mathrm{CW}$ & - & - & 100 \\
\hline ISOL@MYRRHA & Protons & $0.6-1$ & $<200$ & Pulsed & $<200 \mu \mathrm{s}$ & $250 \mathrm{~Hz}$ & $<120$ \\
\hline CERN-ISOLDE (test facility) & Protons & 1.4 & $1-2$ & Pulsed & $2.4-40 \mu \mathrm{s}$ & $0.8 \mathrm{~Hz}$ & $1-2.8$ \\
\hline
\end{tabular}


ensures that hot spots will be avoided and constitutes the main subject of the analysis in Section 4 of this paper.

In order to achieve higher release efficiencies, the second requirement is to reduce the characteristic diffusion length of isotopes out of the liquid-metal. This is ensured in the present concept by decreasing the size of the LBE matrix out of which nuclides have to diffuse. Irradiated LBE will be spread into a shower of droplets with radii in the range of few $100 \mu \mathrm{m}$, which represents a decrease in the characteristic diffusion length of isotopes of two orders of magnitude compared to static-bath ISOLDE liquid-metal targets. Indeed, each of the small droplets constitutes the matrix out of which isotopes will diffuse and, the smaller the droplets size, the faster the diffusion.

The shape of the LBE matrix also influences the characteristic diffusion length of isotopes, and the sphere is the ideal shape for fast release $[6,15]$. Therefore, the present loop-type target aims at producing spherical droplets. Applying the Young-Laplace law [16], we derived that spherical droplets will be produced to meet the following condition:

$r \ll \sqrt{\frac{\gamma}{g\left(\rho_{\text {liq }}-\rho_{\text {vap }}\right)}}$

where $r$ is the spherical-droplet radius, $\gamma$ is the surface tension of LBE, $\rho_{\text {liq }}$ and $\rho_{\text {vap }}$ are the respective densities of liquid and vapor LBE, respectively, while $g$ is the gravity. At the pressure of the diffusion chamber $\left(p=5 \times 10^{-7} \mathrm{mbar}\right)$, the produced droplets will be spherical if their radii are much smaller than $2 \mathrm{~mm}$. Already for $200-\mu \mathrm{m}$ radii droplets this condition is met with a $1 \%$ maximum deviation of the droplet-surface mean curvature from the ideal spherical curvature.

The following sections present the methodology applied for the target design and the optimization process to meet the abovementioned requirements.

\section{Methodology}

Computational Fluid Dynamics (CFD) tools are commonly used for the thermal and hydraulic design of facilities operating with liquidmetal, e.g., MYRRHA [17], ESS [18] and EURISOL [8,19]. Furthermore, important aspects of CFD analysis such as the turbulence modeling, pressure field and pressure drop in a liquid-metal flow have been validated in the framework of thermal-hydraulics design of the MEGAPIE LBE target $[8,19]$. These tools are less expensive and faster to implement for a design-optimization procedure than prototyping each optimization step. Fluent (ANSYS, Canonsburg, PA, USA), has been used for the work reported in this article. Full-scale prototyping and offline tests, following the design study have to be conducted to check particular aspects of the design work presented in this paper.

All the modeled CFD geometries comprise a half-symmetry of the target geometry about the $X Y$-plane (see Fig. 2). This assumption is explained by the fact that the distributions of evacuation and feeder apertures are symmetric with respect to the vertical mid-plane of the flow inlet. The geometries were also restricted to the fluid domain for CFD analysis and the fluid-container interaction has been modeled by adequate "no slip wall" boundary conditions. More than 22,000,000 cells are required to get meshindependent results, e.g., in the half-symmetry distributed-inlet concept 5 discussed in Section 4.3 of this paper. The thermal effects of the proton-beam impact have not been taken into account in these calculations. This simplifies and speeds up calculations during the hydro-dynamical optimization process. Issues related to LBE-temperature profile, heat deposition and dissipation, potential occurrence of pressure waves and resonance are outside the scope of the present paper. While these effects are

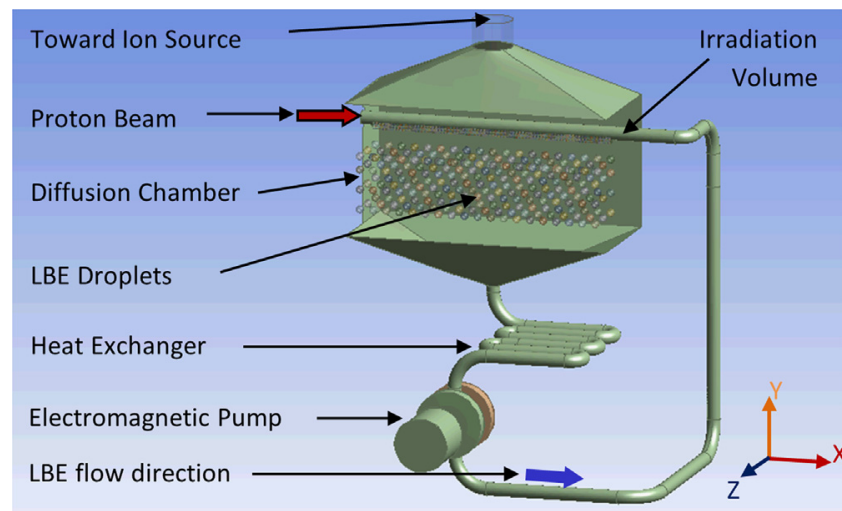

Fig. 2. Initial sketch of the liquid-LBE loop with the irradiation volume of concept 1.

likely to be of concern at highly-pulsed beam facilities, EURISOL is foreseen to operate with a CW proton beam. Given the high pulse repetition rate and long pulse length (see Table 1), the instantaneous power densities will be rather low at ISOL@MYRRHA as well.

Previous liquid-metal target design calculations with $k-\varepsilon$ and $k-\omega$ Shear Stress Transport (SST) turbulence models have shown good agreement with experimental data (mercury target experiment for EURISOL and lead-bismuth for MEGAPIE) for averageflow behavior and mean-velocity fields [20,21]. Because these models are less computationally intensive and since no energymixing process is accounted for in this design-optimization process, the SST $k-\omega$ turbulence model has been used for these steady-state simulations.

\section{Results and discussion}

\subsection{Starting-case geometry of the irradiation volume}

Based on the conceptual view presented in Fig. 1, the startingcase geometry presented in Fig. 2 was used for LBE-dynamics simulation inside the irradiation volume. In this initial concept, the irradiation volume of the target consists of a $20-\mathrm{cm}$ long and $1-\mathrm{cm}$ diameter cylinder. This diameter is selected to accommodate three standard deviations $(3 \sigma)$ of the proton-beam transverse Gaussian profile (FWHM $3.5 \times 3.5 \mathrm{~mm}^{2}$ at the test facility). The cylindrical irradiation volume and the proton-beam direction (Figs. 2 and 3) are coaxial. LBE flows into the irradiation volume through its base section and is evacuated through a series of apertures uniformly distributed over the lower half of the cylinder lateral surface. LBEflow direction is indicated in Fig. 2. This concept, called concept 1 , features 2500 evacuation apertures of $200-\mu \mathrm{m}$ radii, numbers deriving from the fast evacuation (within $100 \mathrm{~ms}$ ) requirement and the objective of reaching the proper regime of droplet formation (the jetting regime) [22].

Considering the fact that the manufacturing of the exit apertures on a cylindrical surface is quite challenging, an alternative concept featuring the evacuation apertures on a flat surface (see A-A section in Fig. 4) was also analyzed. In order to accommodate $3 \sigma$ for the proton beam, the cross-section area and flow rate in the irradiation volume of concept 2 are, correspondingly, twice the values in concept 1 . The geometry of the irradiation volume in concept 2 consists of the half of a $2-\mathrm{cm}$ diameter cylinder. Because the flow rate is doubled, 5000 evacuation apertures are required in order to keep the formation of droplets in the same regime (the jetting regime). Also, due to the larger irradiation volume, the average concentration of short-lived isotopes is half of that in concept 1 . This affects the isotopes-diffusion efficiency. 


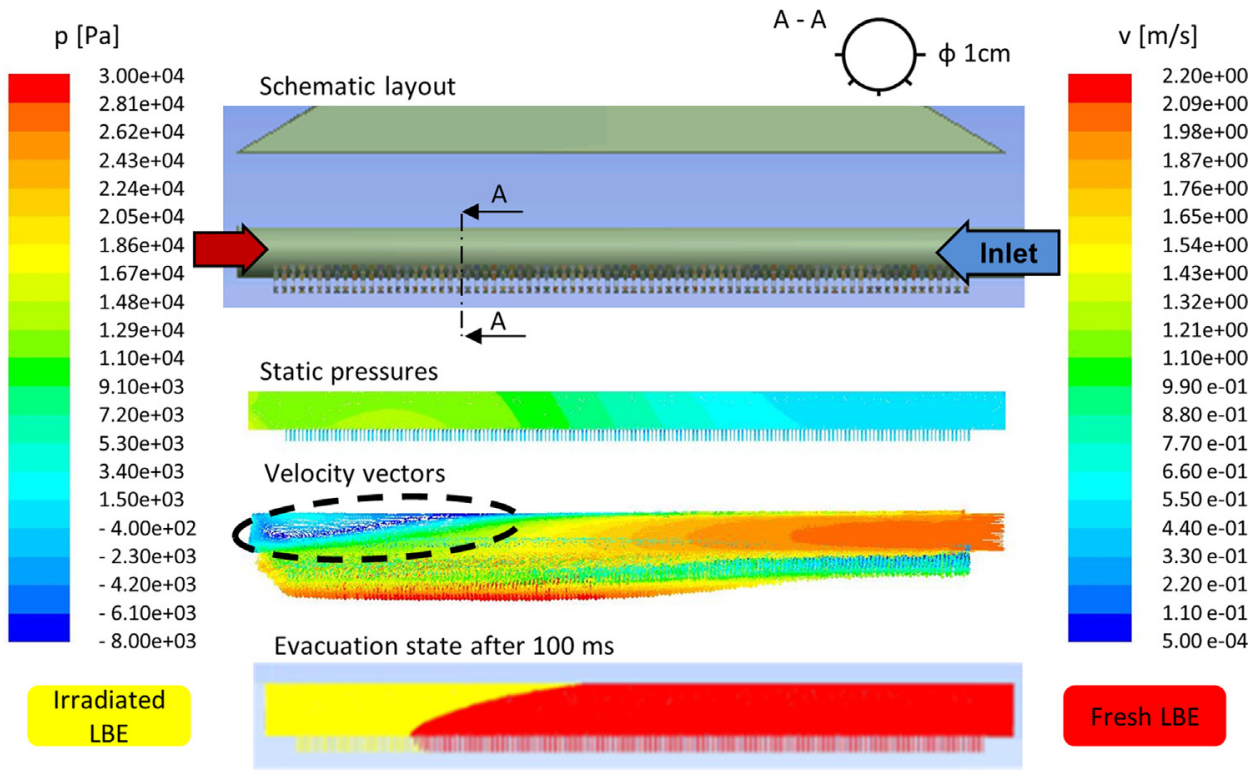

Fig. 3. Schematic layout and CFD-analysis results (on symmetry plane) of the target design in concept 1.

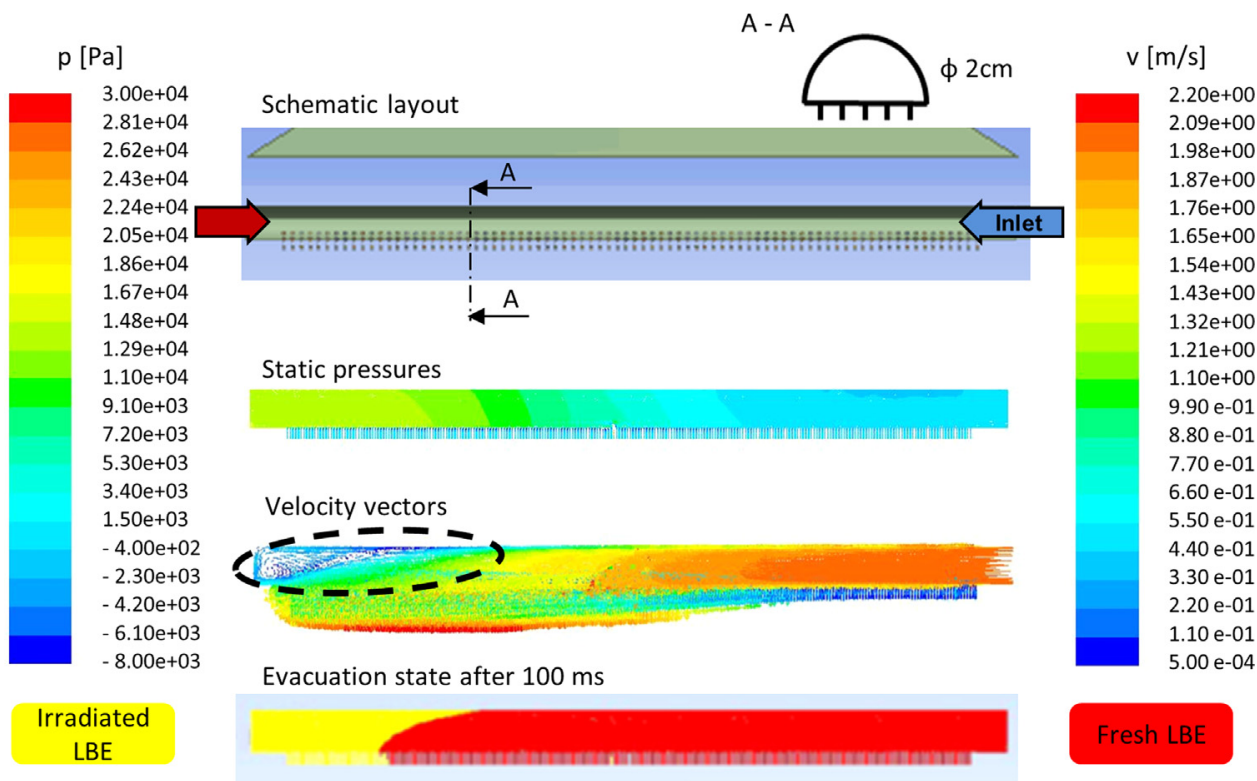

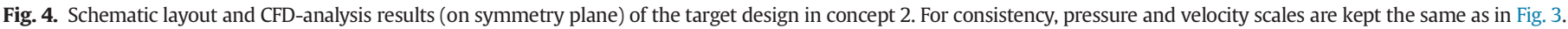

The computed static-pressure distributions of concept 1 (Fig. 3) and concept 2 (Fig. 4) show very low pressure levels in the LBE inside the evacuation apertures on the inlet side of the irradiation volume. Some of these low-pressure regions have values below experimental values of the LBE saturation vapor pressure reported in literature: $10^{-6} \mathrm{~Pa}$ at $500 \mathrm{~K}[7,23]$. This poses a risk of flowinduced cavitation and erosion damage to the target structure. Evidence of cavitation erosion in a pulsed-beam liquid lead target has been reported for ISOLDE target containers [24]. While the origin of the erosion in these targets was not flow, the risk of cavitation damage in this design should be mitigated to assure sufficient target life. Indeed, cavitation can have the effect of limiting the target container lifetime and can even lead to its failure. The envisaged lifetime of the prototype at ISOLDE is of the order of 1-2 weeks while EURISOL is foreseen to operate a target for 3 weeks and ISOL@MYRRHA for 4-8 weeks.

These low-pressure zones are explained by the fact that highpressure gradients are required to bend the high-momentum LBE streamlines through the first apertures on the inlet side. The pressure values computed inside the irradiation volume at the inlet were low enough to induce virtually no flow through the corresponding evacuation apertures (see velocity-vectors plots in Figs. 3 and 4), which also means no droplet formation through these apertures. Besides, pressure progressively builds up inside the irradiation chamber in both concepts while the average horizontal velocity decreases from right to left in Figs. 3 and 4. This is due to the fact that part of the inlet LBE flow is progressively lost through the evacuation apertures, and this leads to a lower flow velocity since the cross-section of the flow volume is constant along the irradiation volume.

The negative values on the pressure scales are not related to the high-momentum-streamline bending effect described earlier. Because of the stream-wise pressure build-up mentioned in the previous paragraph, an acceleration of LBE at the entrance in evacuation apertures, mostly on the left-hand side of the velocity plots (Figs. 3 and 4), led as well to computation of negative pressure values in the corresponding regions. Computation of these, however, unphysical negative pressure values occurs because cavitation effects were 
not modeled. Modeling these effects is not needed at this stage and would increase the computation time.

Recirculation zones were also noticed at the stream-wise end of the irradiation volume in both concepts because of the sudden direction change of the geometry at this end. These zones are marked by the dashed-line contours in Figs. 3 and 4. When they occur in the irradiation volume, these recirculation zones carry produced isotopes, including short-lived ones, for extended periods of time, which leads to decay losses. For this reason, the effect of recirculation zones was studied by computing, for each concept, the portion of irradiated LBE evacuated from the irradiation volume within $100 \mathrm{~ms}$ after the arrival of a proton pulse. It is shown on the evacuation-state plots (Figs. 3 and 4) that $100 \mathrm{~ms}$ after the arrival of a proton pulse, portions of LBE in the recirculation zones have not been evacuated from the irradiation volume to the diffusion chamber. The turbulent mixing region at the interface between fresh and irradiated LBE is not shown on these plots since it spans over a narrow region and does not significantly affect the appreciation of the evacuation state. Throughout the paper, a $50 \%$ threshold value is used for construction of the two-level scale evacuation-state plots.

Recirculation zones in the irradiation volume are to be avoided in order to (1) reduce as much as possible decay losses of shortlived isotopes inside the irradiation volume; (2) prevent occurrence of hot spots and (3) ensure a proper cooling of the beam window at high proton beam power.

In summary, the main issues detected for these two concepts are as follows:

- the presence of very low pressure zones,

- a non-uniform distribution of evacuation velocity-vectors along the proton-beam direction, and

- recirculation of LBE inside the irradiation volume.

Consequently, these simple concepts cannot be used. The design optimization that will be discussed in the following sections considered successively-improved concepts to overcome these issues. From this point on, the half-cylinder design of the irradiation volume in concept 2 has been dropped because its results have not shown any specific advantage to compensate for its major disadvantages:
- larger irradiation-chamber volume,

- higher flow rate i.e. it requires more power at the pump (pressure drops being of the same order as in concept 1), and

- more evacuation apertures per unit surface meaning higher cost for manufacturing and higher risks of merging between closer LBE jets.

\subsection{Transverse-inlet irradiation volume}

Concept 3 (see Fig. 5), features a cylindrical irradiation volume with two inlets perpendicular to the proton-beam axis. The irradiation volume is a $20-\mathrm{cm}$ long and $1-\mathrm{cm}$ diameter cylinder, like in concept 1 . Hence, it requires the same number of evacuation apertures. Since each of the two inlets has the same cross-section area as the inlet in concept 1 , the inlet velocity in concept 3 is half of that in concept 1.

The use of transverse inlets solves the low-pressure regions observed in concepts 1 and 2 . Indeed, it suppresses the need to bend streamlines over small radii of curvature at inlet. Results are presented in Fig. 5. A more uniform distribution of pressure and evacuation-velocity vectors is observed.

However, there were very few negative-pressure regions determined in this case, which are related to the acceleration of LBE at the entrance of few evacuation apertures. This indicates that the distribution of the evacuation-velocity vectors is still not uniform enough. Additionally, extensive recirculation zones are noticed (see dashed-line boxes in Fig. 5). As shown in the state-of-evacuation plot of Fig. 5, the presence of these recirculation zones inside the irradiation volume leads to slow evacuation of part of the irradiated LBE. This issue is dealt with in the next sections through more elaborate concepts.

\subsection{Distributed-inlet irradiation volume}

At this point, LBE recirculation in the irradiation volume is the main issue left to be solved in order to meet the 100-ms evacuation requirement. For this reason, every concept in the following sections is designed with a feeder volume (on top of the irradiation volume) that will host the recirculation zones and thereby prevent their occurrence inside the irradiation volume. In

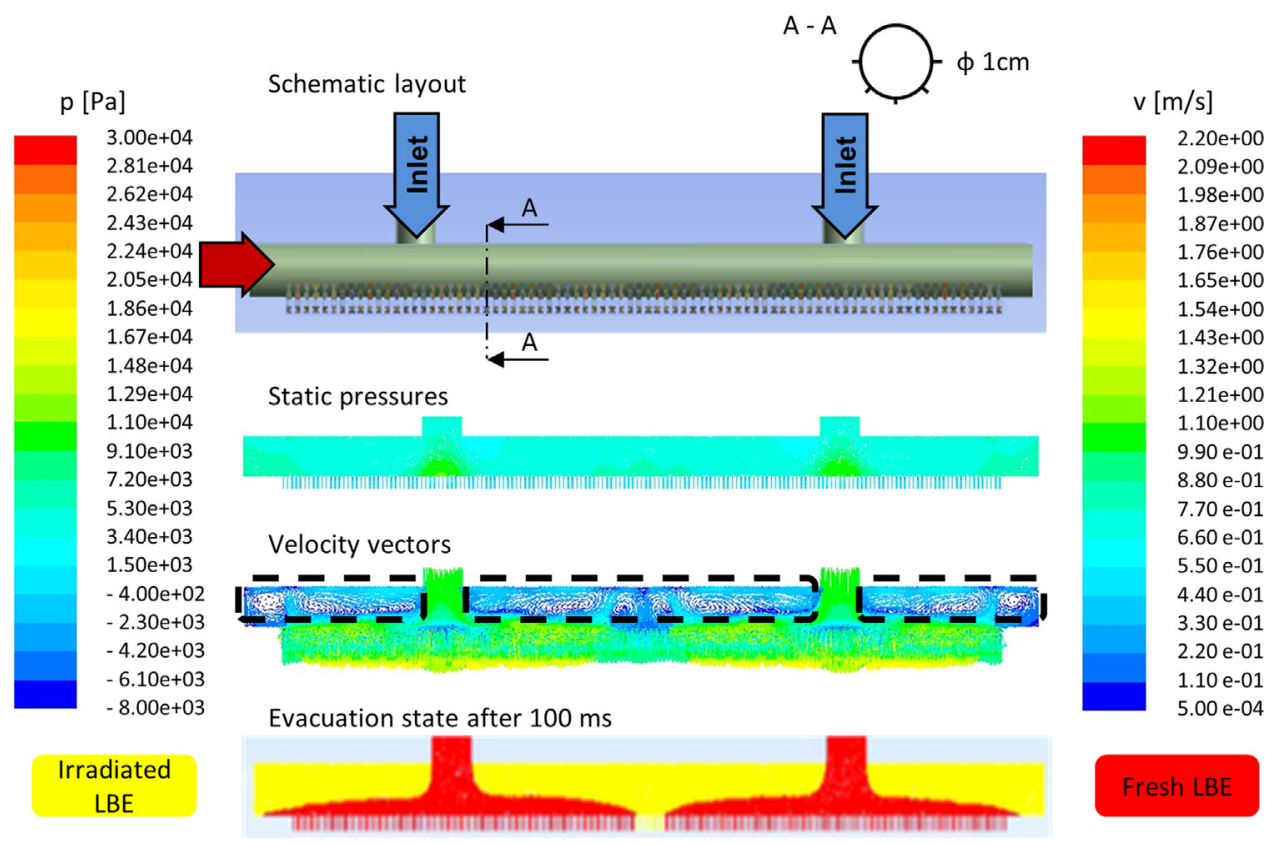

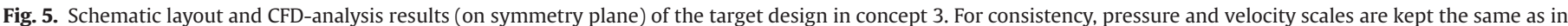
Fig. 3. 
concepts 4 and 5 (Figs. 6 and 7), the double-inlet design of concept 3 is maintained. The inlet direction is also kept normal to the proton-beam direction, but the inlets feed the irradiation volume through the feeder volume. Feeder grids are also foreseen between the inlets and the irradiation volume. These feeder grids are designed to oppose sufficient resistance to the inlet jets in order to uniformly distribute the LBE over the irradiation volume. This is accomplished through the use of feeder-grid apertures with small diameters.

In concept 4 , a single feeder volume with a feeder grid is used. Under these conditions, preliminary simulations results (not presented here) show that feeder-grid apertures with radii $\leq 100-\mu \mathrm{m}$ are required in order to obtain a uniform distribution over the irradiation volume. This results in a feeder grid equipped with 10,000 apertures of $100 \mu \mathrm{m}$ and a feeder volume of $5 \mathrm{~cm}$ in height. Besides, to accommodate the flat feeder grid, the geometry of the irradiation volume consists of the lower half of a $20-\mathrm{cm}$ long and 1-cm diameter cylinder plus a $20-\mathrm{cm} \times 1-\mathrm{cm} \times 0.5-\mathrm{cm}$ parallelepiped on top.
Another concept studied (concept 5) presents two feeder grids and two feeder volumes. Each of these feeder grids is equipped with 2500 apertures of $200-\mu \mathrm{m}$ radii and each of the feeder volumes is $2-\mathrm{cm}$ high. The irradiation-volume geometry is the same as for concept 4 . In both cases (concepts 4 and 5), 1-mm thick feeder grids are used in the computation.

The results of the CFD-analysis for concepts 4 and 5 are presented in Figs. 6 and 7, respectively. The static pressure plots do not display any negative pressure region. In both cases, pressure differences vertically across the feeder grids are mostly constant along the proton-beam axis. This explains the uniform distribution of velocity-vectors, obtained inside the irradiation volume and at evacuation in the diffusion volume. Even though large recirculation zones have been computed (see dashed-line boxes), these have been held in the feeder volumes. Recirculation zones occurring in the feeder volume do not affect the fast evacuation of short-lived radioactive isotopes and they do not present a risk for occurrence of hot spots. Indeed, the feeder
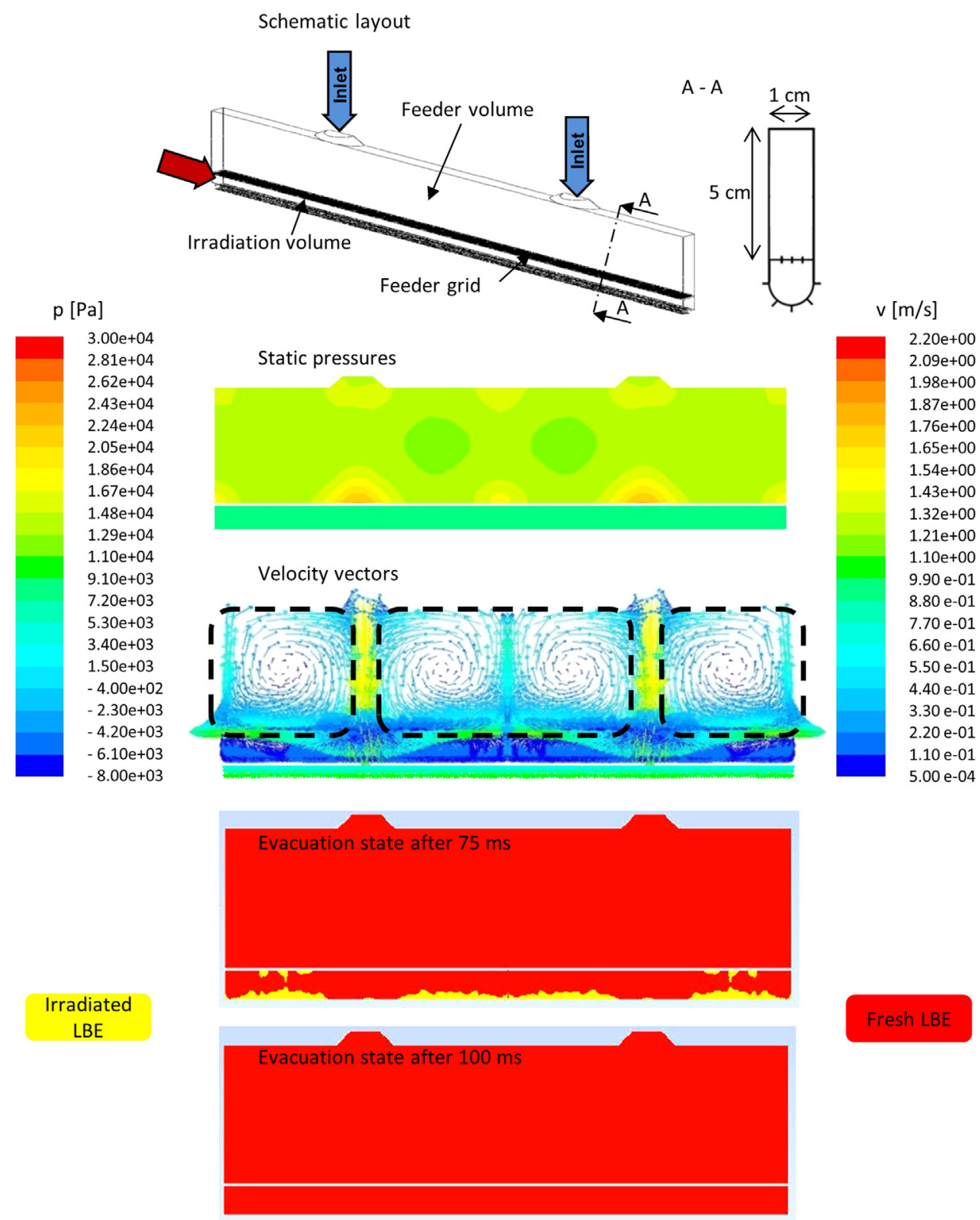

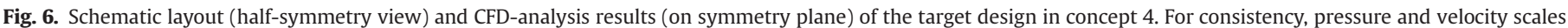
are kept the same as in Fig. 3. 
Schematic layout

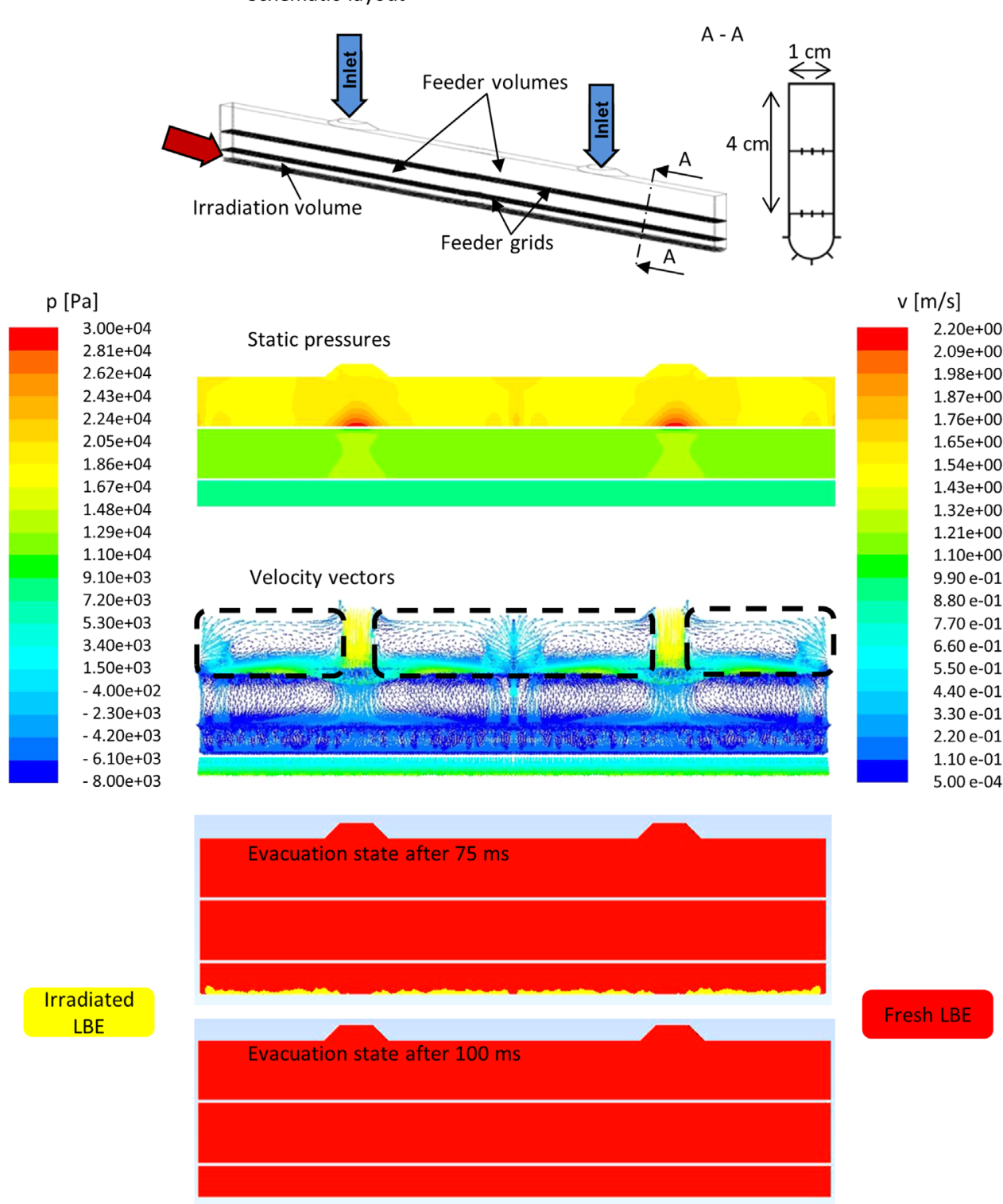

Fig. 7. Schematic layout (half-symmetry view) and CFD-analysis results (on symmetry plane) of the target design in concept 5 . For consistency, pressure and velocity scales are kept the same as in Fig. 3.

volumes are not subject to direct and intensive irradiation by the proton beam.

As a result of the absence of recirculation zones inside the irradiation volume, in both concepts 4 and 5 all the irradiated LBE is evacuated from the irradiation volume within $100 \mathrm{~ms}$ after a proton-pulse impact. These two acceptable solutions however imply some degrees of complexity for the manufacturing, with either a doublet of feeder grids or smaller feeder-grid apertures $(100 \mu \mathrm{m}$ instead of $200 \mu \mathrm{m})$.

\subsection{Prism-shape feeder volume}

The prism-shape feeder volume concept presented here is used as an alternative, to reduce the complexity of manufacturing mentioned in Section 4.3 for concepts 4 and 5. It allows for larger inlets and consequently lower inlet velocity to provide the same flow rate of LBE as in concepts 4 and 5 . The required inlet velocity in this concept is almost reduced by an order of magnitude compared to previous concepts, thereby reducing the strength of the inlet-jet effect. The difference between the momentum of LBE in the inlet jet and LBE in the rest of the feeder volume is thus reduced. With such a reduced inlet-jet, the design of the feeder grid can be relaxed and one single feeder grid of $200-\mu \mathrm{m}$ radii apertures is necessary to obtain a uniform distribution of velocity vectors through the irradiation volume and at evacuation (Fig. 8). 2500 Apertures are hosted on the feeder grid which has a thickness of $1 \mathrm{~mm}$, similar to concepts 4 and 5 .

Results of the CFD analysis are included in Fig. 8: velocityvectors are uniformly distributed in the irradiation volume and at evacuation to the diffusion volume. No recirculation zone occurs in the irradiation volume and the design meets the 100-ms evacuation requirement.

\subsection{Discussion}

Unforeseen issues have been revealed through simulations of the starting-case geometries proposed for the irradiation volume. The available amount of data resulting from these initial simulations 
Schematic layout
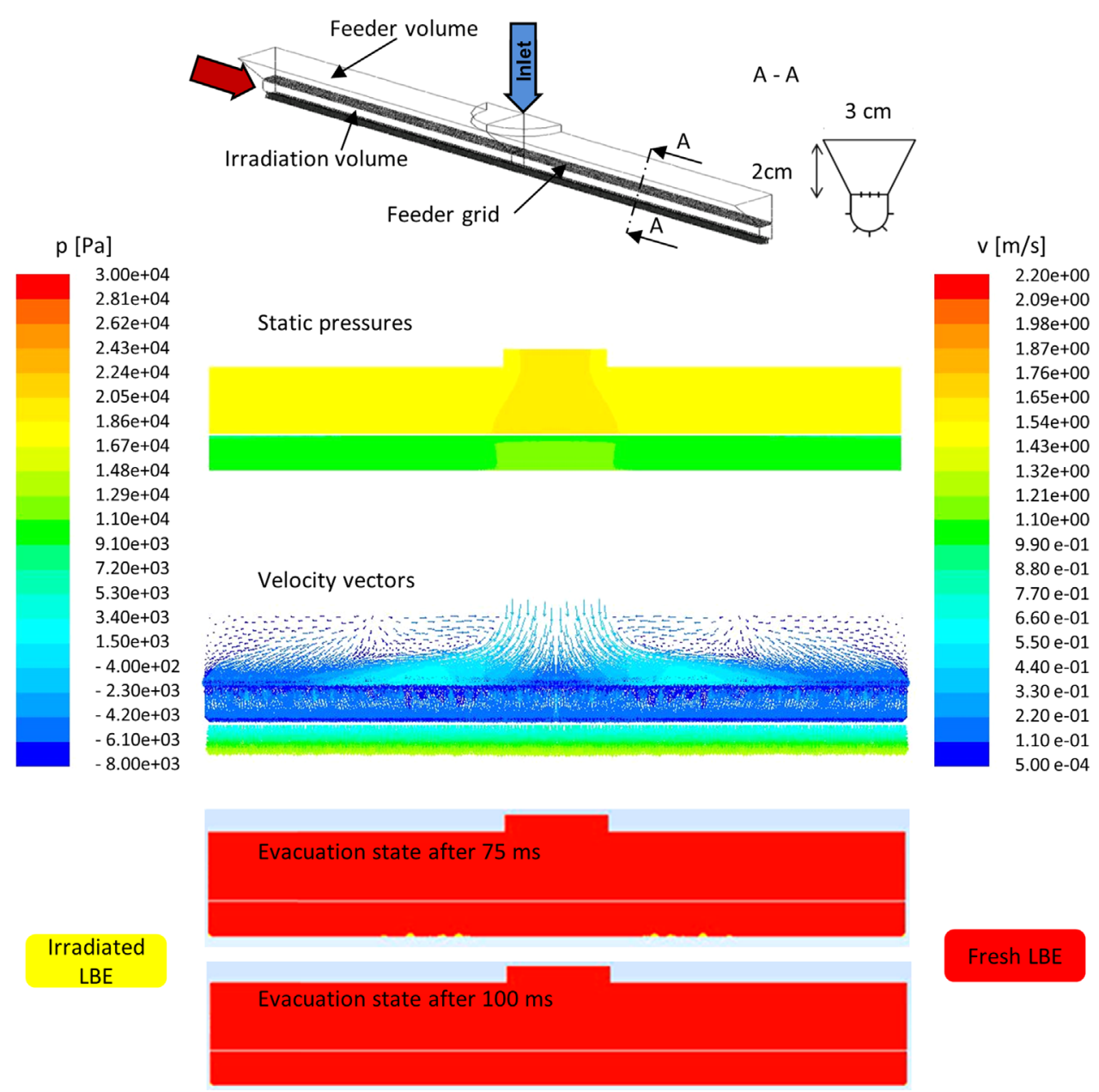

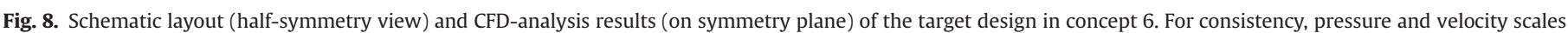
are kept the same as in Fig. 3.

provided a unique insight on the causes and potential solutions of these issues. As a result, a key feature of the optimized concepts is the need for a set of feeder volumes and feeder grids.

Thin feeder grids have been foreseen in the current design since this reduces the pressure drop across the grids. However, the uniform flow computed is not negatively affected by the use of thicker feeder grids that may be required for the LIEBE prototype in order to make the design more resistant to pressure waves generated by the pulsed proton beam at the test facility. Thicker feeder grids will simply have the effect of increasing the pressure drops.

Diffusion coefficients typically show an exponential dependence on temperature and the release of isotopes is more efficient at higher temperature. For this reason, the satisfactory concepts (4-6) have been simulated with LBE data at different temperatures ranging from $500 \mathrm{~K}$ to $1200 \mathrm{~K}$. It results from these calculations that the uniform flow of LBE in the irradiation volume is maintained over this range of temperatures for concepts 5 and 6 but not for concept 4 . This can be explained since LBE viscosity decreases by more than a factor of two with increased temperature over this range $(0.00223 \mathrm{~Pa} \cdot \mathrm{s}$ at $500 \mathrm{~K}$ and $0.000926 \mathrm{~Pa} \cdot \mathrm{s}$ at $1200 \mathrm{~K}$ [23]). The lower viscosity reduces the dissipation of the inlet-jets energy to adjacent LBE by momentum diffusion. For temperatures higher than $600 \mathrm{~K}$ this effect disrupts the uniform flow of LBE in concept 4.

The use of smaller feeder-grid apertures $(100-\mu \mathrm{m}$ radii) in concept 4 makes it more prone to clogging of the feeder grids than concepts 5 and 6 . The effect of such a clogging of the feeder grid, due to impurities carried on by the inlet flow of LBE, has been assessed. Simulations were run for the three concepts (4-6) with a partial clogging of the feeder-grid apertures. Assuming the impurities are brought in with the inlet stream, only the first feeder grid of concept 5 was partially clogged while for concepts 4 and 6 the single grid was partially clogged in the simulation. In all the concepts the clogged apertures were simulated in line with the inlets. Clogging of apertures was modeled by suppressing the apertures over a compact portion of the feeder-grid (i.e. replacing them by a volume representing a metal piece). The results have shown that even with 30\% clogging of its first feeder grid, the uniform flow of LBE in the irradiation volume is maintained for concept 5 . Because concepts 4 and 6 are only equipped with a single feeder grid, the uniform flow of LBE in the irradiation volume as well as the complete LBE evacuation determined in nominal conditions are already disrupted with a $10 \%$ clogging.

Out of the three concepts that meet the preset design requirements, concept 5 proves to be the most robust. However, it is quite demanding in terms of complexity in manufacturing. On the other hand, concept 6 can be made more robust through the use of a filter on the feeder line. These concepts will be prototyped for tests at CERN-ISOLDE, where the available proton beam is of similar energy as foreseen for EURISOL. The beam spot at ISOLDE is also characterized by a standard deviation falling in the range of investigated values for the liquid-metal 100-kW EURISOL direct target [6]. However, the biggest difference is that EURISOL envisages a CW proton beam while 
ISOLDE operates with a pulsed beam. Specific issues unlikely in CW mode and related to the pulsed-beam time structure of ISOLDE will therefore be studied for the prototype.

\section{Conclusion}

The CFD simulations presented in this paper have been carried out in support of the design and optimization processes of a moltenLBE target loop for the production of beams of short-lived radioactive ions at high-power ISOL facilities. A CFD analysis was used since it has been validated in the past with experimental data for liquidmetal flow. This analysis allowed us to avoid a lengthy and costly process of prototyping and testing every investigated design.

The target-design optimization reported here was conducted with the aim of a complete evacuation of the irradiated LBE from the irradiation volume within $100 \mathrm{~ms}$ after the impact of a proton pulse. Three designs meeting this requirement have been developed. In each of these cases the inlet-jet effect was solved with a combination of two approaches: (1) increasing the size of inlet sections in order to reduce inlet velocities; and (2) opposing one or two high-resistance feeder grids to the inlet jet.

With this strategy, within a compact geometry, the jet flow with high-momentum that exists at the inlets is transformed into a uniform flow in the irradiation volume and at evacuation. Issues of low-pressure zones have been solved by avoiding unnecessary bending of the flow inside the compact geometry of the target. In addition, the ideal spherical shape of the droplets is ensured by making small droplets (radii $\ll 2 \mathrm{~mm}$ for LBE). Out of the three concepts that meet the preset design requirements, concept 5 proved to be the most robust with regards to risks of clogging.

\section{Acknowledgments}

This work was partially funded by the BriX network project P7/12 of the Interuniversity Attraction Poles (IAP) programme of the Belgian Science Policy Office.

\section{References}

[1] M. Huyse, The why and how of radioactive-beam research, in: J. Al-Khalili, E. Roeckl (Eds.), The Euroschool Lectures on Physics with Exotic Beams, vol. I, Springer, Berlin, Heidelberg, 2004, p. 1.

[2] Y. Blumenfeld, P. Butler, J. Cornell, G. Fortuna, M. Lindroos, International Journal of Modern Physics E. Nuclear Physics 18 (2009) 1960.
[3] L. Popescu (for the ISOL@MYRRHA and MYRRHA Collaborations), Nuclearphysics applications of MYRRHA, in: Proceedings of the INPC2013 Conference and EPJ Web of Conferences, vol. 66, 2014, p. 10011.

[4] Y. Zhang, G.D. Alton, Nuclear Instruments and Methods: A 521 (2004) 72.

[5] M. Dombsky, P. Bricault, Nuclear Instruments and Methods: B 266 (2008) 4240.

[6] EURISOL Project Coordination Board, Final Report of the EURISOL Design Study (2005-2009), GANIL, France, 2009 (220 pp.).

[7] T.V. Dury, Journal of Nuclear Science and Technology. 41 (2004) 285.

[8] K. Samec, R.Ž. Milenković, S. Dementjevs, M. Ashrafi-Nik, A. Kalt, Nuclear Instruments and Methods: A 606 (2009) 281.

[9] E. Noah, L. Bruno, R. Catherall, J. Lettry, T. Stora, Nuclear Instruments and Methods: B 266 (2008) 4303.

[10] R.Ž. Milenković, S. Dementjevs, K. Samec, A. Flerov, K. Thomsen, Nuclear Instruments and Methods: A 608 (2009) 175.

[11] P. Jung, T. Koppitz, G. Muller, A. Weisenburger, M. Futakawa, Y. Ikeda, Journal of Nuclear Materials 343 (2005) 92.

[12] J. Lettry, R. Catherall, P. Drumm, P. Van Duppen, A.H.M. Evensen, G.J. Focker A. Jokinen, O.C. Jonsson, E. Kugler, H. Ravn, Nuclear Instruments and Methods: B 126 (1997) 130

[13] J. Lettry, R. Catherall, G. Cyvoct, P. Drumm, A.H.M. Evensen, M. Lindroos O.C. Jonsson, E. Kugler, J. Obert, J.C. Putaux, J. Sauvage, K. Schindl, H. Ravn, E. Wildner, Nuclear Instruments and Methods: B 126 (1997) 170.

[14] T. Stora, J. Lettry, R. Catherall, E. Noah, R. Wilfinger, L. Penescu, E. Bouquerel, S. Fernandes, M. Santana-Leitner, L. Zanini, I. Guenter, F. Groeschel, The EURISOL facility: feasibility study for the $100 \mathrm{~kW}$ direct targets, in: An internal task note of the EURISOL Project in: 〈http://www.eurisol.org/site02/doc details. php? url=search_results.php\&operation=download\&docu=450\&type $\rangle, 2006$.

[15] M. Fujioka, Y. Arai, Nuclear Instruments and Methods in Physics Research 186 (1981) 409.

[16] B. Lautrup, Physics of Continuous Matter, Second Edition: Exotic and Everyday Phenomena in the Macroscopic World, Taylor \& Francis, Boca Raton, FL, USA, 2011.

[17] M. Vanderhaegen, J. Vierendeels, B. Arien, Nuclear Engineering and Design 241 (2011) 775.

[18] J. Wolters, G. Hansen, E.M.J. Komen, F. Roelofs, Validation of CFD models with respect to the thermal-hydraulic design of the ESS target, in: Proceedings of the IAEA Technical Meeting, Karlsruhe, Germany, 〈http://www.fz-juelich.de/ cae/servlet/contentblob/930156/publicationFile/12559/ValidationCFDESS.pdf , 2003.

[19] C. Fazio, et al., Nuclear Engineering and Design 238 (2008) 1471.

[20] K. Samec, R.Z. Milenkovic, L. Blumenfeld, S. Dementjevs, C. Kharoua, Y. Kadi Nuclear Instruments and Methods: A 638 (2011) 1.

[21] R. Stieglitz, M. Daubner, A. Batta, C.H. Lefhalm, Nuclear Engineering and Design 237 (2007) 1765.

[22] C. Clanet, J.C. Lasheras, Journal of Fluid Mechanics 383 (1999) 307.

[23] N.E. Agency, Handbook on Lead-Bismuth Eutectic Alloy and Lead Properties, Materials Compatibility, Thermal-hydraulics and Technologies, OECD Publications, France, 2007.

[24] J. Lettry, G. Arnau, M. Benedikt, S. Gilardoni, R. Catherall, U. Georg, G. Cyvogt, A. Fabich, O. Jonsson, H. Ravn, S. Sgobba, G. Bauer, H. Brucherstseifer, T. Graber, C. Güdermann, L. Ni, R. Rastani, Nuclear Instruments and Methods: B 204 (2003) 251 . 